\title{
Goldstone-Type Pseudoscalar Mesons: Instantaneous Bethe-Salpeter Models
}

\author{
Wolfgang Lucha ${ }^{1, a}$ \\ ${ }^{1}$ Institute for High Energy Physics, Austrian Academy of Sciences, Nikolsdorfergasse 18, A-1050 Vienna, \\ Austria
}

\begin{abstract}
A nontrivial instantaneous variant of the Bethe-Salpeter formalism allows us to discuss massless pseudoscalar mesons from a configuration-space-potential point of view.
\end{abstract}

\section{Incentive: pions and kaons as Goldstonic quark-antiquark bound states}

Quantum field theory describes bound states of two fermions, of particle and relative momenta $p_{1,2}$ and $p$, ignoring their total momenta, by Bethe-Salpeter amplitudes $\Phi(p)$ solving Bethe-Salpeter equations

$$
\Phi(p)=\frac{\mathrm{i}}{(2 \pi)^{4}} S_{1}\left(p_{1}\right) \int \mathrm{d}^{4} q K(p, q) \Phi(q) S_{2}\left(-p_{2}\right)
$$

defined by kernels $K(p, q)$ subsuming the underlying interactions, and fermion propagators $S_{1,2}(p)$, by Lorentz covariance given by mass and wave-function renormalization functions $M_{1,2}\left(p^{2}\right)$ and $Z_{1,2}\left(p^{2}\right)$ :

$$
S_{1,2}(p)=\frac{\mathrm{i} Z_{1,2}\left(p^{2}\right)}{\not p-M_{1,2}\left(p^{2}\right)+\mathrm{i} \varepsilon}, \quad \not p \equiv p^{\mu} \gamma_{\mu}, \quad \varepsilon \downarrow 0 .
$$

Potentials from inversion [1] offer an intuitive view at light pseudoscalar mesons as quark bound states and Goldstone bosons due to spontaneous chiral symmetry breaking of quantum chromodynamics [2].

\section{Instantaneous bound-state equation remembering Bethe-Salpeter origin}

Already some time ago, in an attempt to retain in the resulting three-dimensional formalism as much as conceivable of all the information of dynamical origin encoded in the propagators (2) of the considered bound-state constituents, we proposed a kind of (relativistically) least-damage instantaneous reduction of the Bethe-Salpeter framework [3]. Within our approach, we achieve this objective not by setting the propagator functions $M\left(p^{2}\right)$ and $Z\left(p^{2}\right)$ as a whole equal to constants, no longer aware of any dynamical effects. Rather, we only discard any dependence of the propagator functions on the time component $p_{0}$ of the relative momentum $p$. In other words, when dealing with $M\left(p^{2}\right)$ and $Z\left(p^{2}\right)$, we place, tentatively, our wagers on some type of " $p_{0}^{2}=0$ approximation." In this case, and under the equal-time assumption that all interactions incorporated by their integral kernel $K(p, q)$ are instantaneous - tantamount to the requirement that $K(p, q)$ does not depend on the time components of the relative momenta $p$ and $q$, i.e., $K(p, q)=K(\boldsymbol{p}, \boldsymbol{q})$ - , the Bethe-Salpeter equation (1) can be integrated with respect to $p_{0}$; the result of the integration is a three-dimensional bound-state equation governing the so-called Salpeter amplitude

\footnotetext{
ae-mail: Wolfgang.Lucha@oeaw.ac.at
} 


$$
\phi(\boldsymbol{p}) \propto \int \mathrm{d} p_{0} \Phi(p) .
$$

Expressed by use of one-fermion free energies $E_{1,2}(p)$ and positive/negative-energy projectors $\Lambda_{1,2}^{ \pm}(\boldsymbol{p})$,

$$
E_{1,2}(p) \equiv \sqrt{p^{2}+M_{1,2}^{2}\left(p^{2}\right)}, \quad \Lambda_{1,2}^{ \pm}(\boldsymbol{p}) \equiv \frac{E_{1,2}(p) \pm \gamma_{0}\left[\boldsymbol{\gamma} \cdot \boldsymbol{p}+M_{1,2}\left(p^{2}\right)\right]}{2 E_{1,2}(p)}, \quad p \equiv|\boldsymbol{p}|,
$$

for a bound state of spin- $\frac{1}{2}$ fermion and spin- $\frac{1}{2}$ antifermion, with relative momentum $\boldsymbol{p}$ and bound-state mass $\widehat{M}$, our instantaneous Bethe-Salpeter equation [3] is, in the center-of-momentum frame, given by

$$
\phi(\boldsymbol{p})=Z_{1}\left(p^{2}\right) Z_{2}\left(p^{2}\right)\left(\frac{\Lambda_{1}^{+}(\boldsymbol{p}) \gamma_{0} I(\boldsymbol{p}) \Lambda_{2}^{-}(\boldsymbol{p}) \gamma_{0}}{\widehat{M}-E_{1}(p)-E_{2}(p)}-\frac{\Lambda_{1}^{-}(\boldsymbol{p}) \gamma_{0} I(\boldsymbol{p}) \Lambda_{2}^{+}(\boldsymbol{p}) \gamma_{0}}{\widehat{M}+E_{1}(p)+E_{2}(p)}\right), \quad p \equiv|\boldsymbol{p}|,
$$

abbreviating, for ease of notation, the three-dimensional remainder of the interaction term in Eq. (1) by

$$
I(\boldsymbol{p}) \equiv \frac{1}{(2 \pi)^{3}} \int \mathrm{d}^{3} q K(\boldsymbol{p}, \boldsymbol{q}) \phi(\boldsymbol{q}) .
$$

The comparison with predictions obtained upon enabling the analytic integrability of the instantaneous limit of Eq. (1) with respect to $p_{0}$ by approximation of both propagators $S_{1,2}$ by just their free forms [4] shows that describing bound states of quarks [5] by Eq. (3) has visible impact on their mass spectra [6].

\section{Configuration-space interquark potential: straightforward determination}

In principle, within the framework of Sec. 2 the way how to extract underlying interquark potentials by inversion is pretty clear. For simplicity, let's focus on bound states composed of particle and associated antiparticle, so that the indices 1, 2 may be dropped. In this case, our bound-state equation (3) becomes

$$
\phi(\boldsymbol{p})=Z^{2}\left(p^{2}\right)\left(\frac{\Lambda^{+}(\boldsymbol{p}) \gamma_{0} I(\boldsymbol{p}) \Lambda^{-}(\boldsymbol{p}) \gamma_{0}}{\widehat{M}-2 E(p)}-\frac{\Lambda^{-}(\boldsymbol{p}) \gamma_{0} I(\boldsymbol{p}) \Lambda^{+}(\boldsymbol{p}) \gamma_{0}}{\widehat{M}+2 E(p)}\right), \quad p \equiv|\boldsymbol{p}| .
$$

For each pseudoscalar meson that is a bound state built up by a quark and corresponding antiquark, its Salpeter amplitude $\phi(\boldsymbol{p})$ can be cast, in terms of two ${ }^{1}$ independent components $\varphi_{1,2}(\boldsymbol{p})$, into the form

$$
\phi(\boldsymbol{p})=\left[\varphi_{1}(\boldsymbol{p}) \frac{\gamma_{0}\left[\boldsymbol{\gamma} \cdot \boldsymbol{p}+M\left(p^{2}\right)\right]}{E(p)}+\varphi_{2}(\boldsymbol{p})\right] \gamma_{5}, \quad E(p) \equiv \sqrt{p^{2}+M^{2}\left(p^{2}\right)}, \quad p \equiv|\boldsymbol{p}| .
$$

The kernel, $K(\boldsymbol{p}, \boldsymbol{q})$, comprises Lorentz nature and momentum dependence of the effective interactions between the bound-state constituents; assuming identical couplings to quark and antiquark, in terms of generalized Dirac matrices $\Gamma$ and associated Lorentz-scalar potentials $V_{\Gamma}(\boldsymbol{p}, \boldsymbol{q})$, its action on $\phi(\boldsymbol{q})$ reads

$$
K(\boldsymbol{p}, \boldsymbol{q}) \phi(\boldsymbol{q})=\sum_{\Gamma} V_{\Gamma}(\boldsymbol{p}, \boldsymbol{q}) \Gamma \phi(\boldsymbol{q}) \Gamma .
$$

We fix the Dirac nature by use of the Fierz-symmetry-enforcing ${ }^{2}$ linear combination of tensor products

$$
\Gamma \otimes \Gamma=\frac{1}{2}\left(\gamma_{\mu} \otimes \gamma^{\mu}+\gamma_{5} \otimes \gamma_{5}-1 \otimes 1\right) .
$$

\footnotetext{
${ }^{1}$ Pseudoscalar mesons are states of zero spin and relative orbital angular momentum; by inspection of the charge-conjugation and parity quantum numbers related, in the decomposition over a complete set of Dirac matrices, to the independent components of the Salpeter amplitude, we realize that only two of the latter are compatible with the expected behaviour of spin-singlet states.

${ }^{2}$ Fierz symmetry is the invariance of Lorentz-scalar quartic products of Dirac field operators $\psi_{1, \ldots, 4}(x)$ under a rearrangement of $\psi_{1, \ldots, 4}(x)$; formulated in terms of Dirac matrices $\Gamma_{i=1, \ldots, 16}, \sum_{i} \bar{\psi}_{1}(x) \Gamma_{i} \psi_{2}(x) \bar{\psi}_{3}(x) \Gamma_{i} \psi_{4}(x)=\sum_{i} \bar{\psi}_{1}(x) \Gamma_{i} \psi_{4}(x) \bar{\psi}_{3}(x) \Gamma_{i} \psi_{2}(x)$.
} 
Assuming the potential $V_{\Gamma}(\boldsymbol{p}, \boldsymbol{q})$ to be of convolution type and to exhibit spherical symmetry enables us to split off all dependence on angular variables. Equation (4) reduces to a set of two, coupled equations (one of which is merely algebraic) for the radial factors $\varphi_{1,2}(p)$ of the independent components of $\phi(\boldsymbol{p})$ :

$2 E(p) \varphi_{2}(p)+2 Z^{2}\left(p^{2}\right) \int_{0}^{\infty} \frac{\mathrm{d} q q^{2}}{(2 \pi)^{2}} V(p, q) \varphi_{2}(q)=\widehat{M} \varphi_{1}(p), \quad 2 E(p) \varphi_{1}(p)=\widehat{M} \varphi_{2}(p), \quad q \equiv|q| ;$ herein, the configuration-space potential $V(r)$ aimed for enters via the radial potential function $V(p, q)$ :

$$
V(p, q) \equiv \frac{8 \pi}{p q} \int_{0}^{\infty} \mathrm{d} r \sin (p r) \sin (q r) V(r), \quad r \equiv|x| .
$$

At the Goldstone point $\widehat{M}=0$ of the arising spectrum of mass eigenvalues $\widehat{M}$, the set decouples and the algebraic equation implies $\varphi_{1}(\boldsymbol{p}) \equiv 0$. Thus, a single bound-state equation determines $\phi(\boldsymbol{p})=\varphi_{2}(\boldsymbol{p}) \gamma_{5}$ :

$$
E(p) \varphi_{2}(p)+Z^{2}\left(p^{2}\right) \int_{0}^{\infty} \frac{\mathrm{d} q q^{2}}{(2 \pi)^{2}} V(p, q) \varphi_{2}(q)=0 .
$$

From the configuration-space representation of this relation, the sought interquark potential follows as

$$
V(r)=-\frac{\widetilde{T}(r)}{\varphi_{2}(r)},
$$

with $\widetilde{T}(r)$ denoting the Fourier transform of the effective kinetic term $E(p) \varphi_{2}(p) / Z^{2}\left(p^{2}\right)$ for $Z\left(p^{2}\right) \neq 0$.

Inverse problems of the type tackled here need, as informational input, solutions of the bound-state equation one is concerned with. In our case, this rôle is filled by $\varphi_{2}(\boldsymbol{p})$, deduced from its ancestor $\Phi(p)$. We may harvest the latter from Dyson-Schwinger analyses of the quark propagator in Euclidean space (identified by underlining) by exploiting the fact [7] that, in the chiral limit, a Ward-Takahashi identity connects full quark propagator and flavour-nonsinglet pseudoscalar-meson Bethe-Salpeter amplitude:

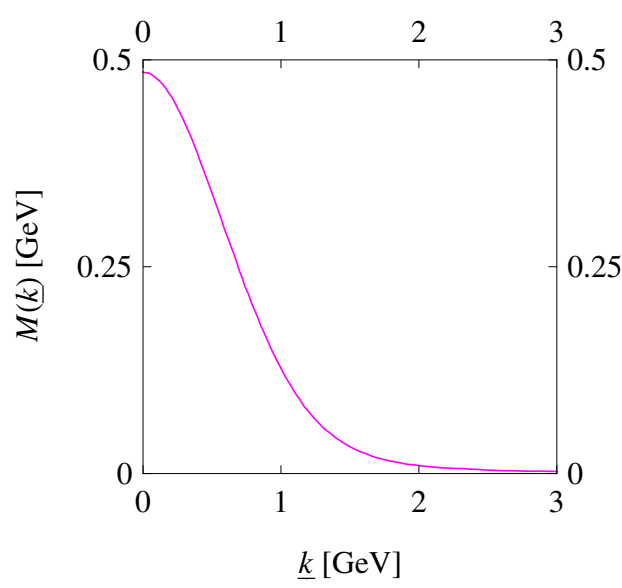

(a)

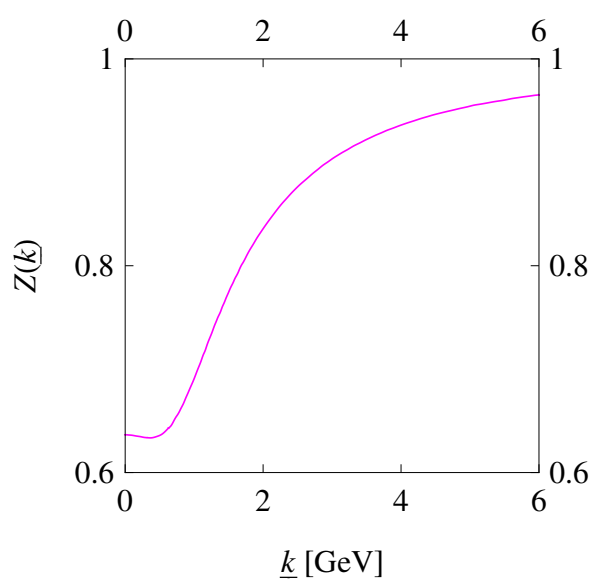

(b)

Figure 1. Quark propagator functions: quark mass function $M(\underline{k})$ (a) and wave-function renormalization $Z(\underline{k})(b)$, plotted as functions of $\underline{k} \equiv\left(\underline{k}^{2}\right)^{1 / 2}$ [9], from the Dyson-Schwinger model for the quark propagator $S(\underline{k})$ of Ref. [8]. 


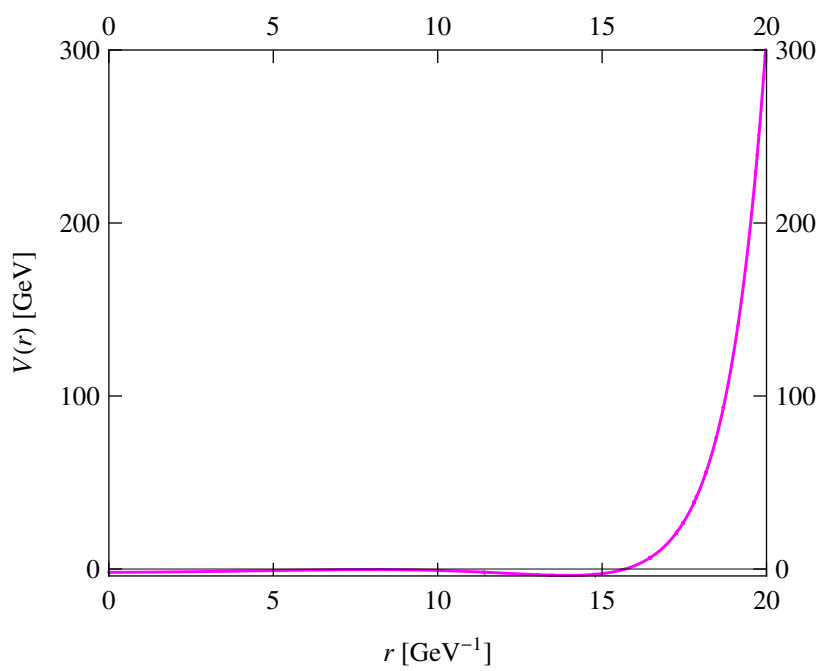

Figure 2. Configuration-space potential $V(r)$ from Fierz-symmetric effective interaction, by inverting the Salpeter amplitude that emerges via Eq. (5) from the quark-propagator solution of the Dyson-Schwinger model of Ref. [8].

$$
\Phi(\underline{k}) \propto \frac{Z\left(\underline{k}^{2}\right) M\left(\underline{k}^{2}\right)}{\underline{k}^{2}+M^{2}\left(\underline{k}^{2}\right)} \underline{\gamma}_{5}+\text { subleading contributions . }
$$

Seeking both $M\left(p^{2}\right)$ and $Z\left(p^{2}\right)$, we are led to the Dyson-Schwinger solutions [8] presented by Ref. [9].

With a well-defined starting point given in form of the quark propagator functions of Fig. 1 at hand, it is just a matter of one's numerical skills to pin down the potential $V(r)$ at least in graphical form [10]. Figure 2 reveals the result: of all insights surely most essential is the square-well shape of the potential.

Some limited insight about the behaviour of the interquark potential can be gained by attributing to the propagator functions $M\left(p^{2}\right)$ and $Z\left(p^{2}\right)$ entering into the three-dimensional bound-state equation (4) some prescribed constant values: approximating the fermion propagators (2) by their free counterparts with effective constituent mass $m$ [11] corresponds to considering the limit $M\left(p^{2}\right) \rightarrow m$ and $Z\left(p^{2}\right) \rightarrow 1$ of the approach of Sec. 2. In this case, the $p_{0}$ integral of the static limit of Eq. (1) may be given anyway. From the solution for $M\left(\underline{k}^{2}\right)$ reported by Ref. [12] (Fig. 3(a)), we derive the potentials $V(r)$ of Fig. 3(b), rising confiningly, for $m$ below some crucial value, from a finite negative value at $r=0$ to infinity [13].

\section{Configuration-space interquark potential: closing in on analytic findings}

Within the free-propagator instantaneous Bethe-Salpeter formalism [14], we are able to prove analytic statements about interquark potentials if we place the focus on specific aspects of the quark propagator. Dyson-Schwinger studies get that $M\left(\underline{k}^{2}\right)$ drops for $\underline{k}^{2} \rightarrow \infty$ roughly like $1 / \underline{k}^{2}$ [12]; axiomatic quantum field theory tells us that quark confinement is ensured by the existence in $M\left(\underline{k}^{2}\right)$ of an inflection point at $\underline{k}^{2}>0[15]$. Both aspects are captured by the ansatz $[16,17]$, with mass $(\mu)$ and mixing $(\eta)$ parameters,

$$
\Phi(\underline{k})=\left[\frac{1}{\left(\underline{k}^{2}+\mu^{2}\right)^{2}}+\frac{\eta \underline{k}^{2}}{\left(\underline{k}^{2}+\mu^{2}\right)^{3}}\right] \underline{\gamma}_{5}, \quad \mu>0, \quad \eta \in \mathbb{R} .
$$




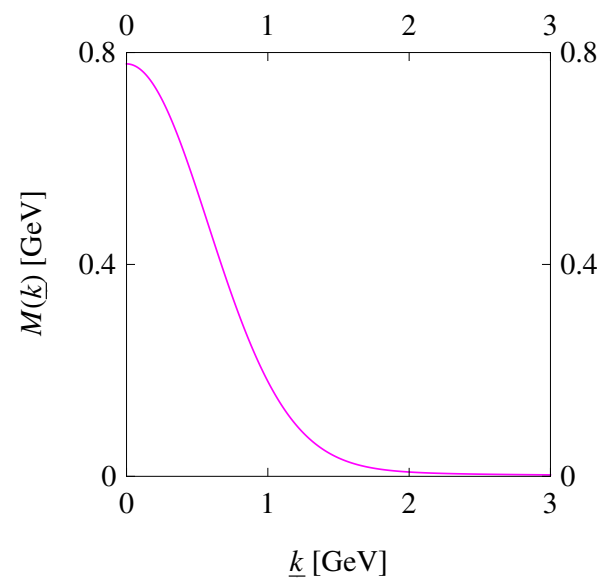

(a)

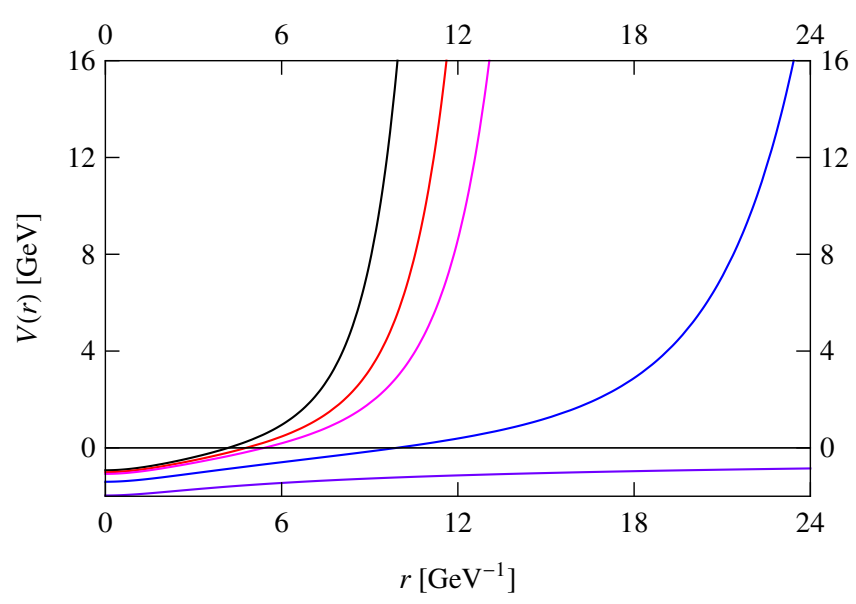

(b)

Figure 3. (a) Mass function $M(\underline{k})$ derived from the Dyson-Schwinger model of Ref. [12] for the quark propagator. (b) Associated configuration-space potential $V(r)$ from Fierz-symmetric kernel $K(\boldsymbol{p}, \boldsymbol{q})$ for constituent quark mass $m=0$ (black), $m=0.35 \mathrm{GeV}$ (red), $m=0.5 \mathrm{GeV}$ (magenta), $m=1 \mathrm{GeV}$ (blue), and $m=1.69 \mathrm{GeV}$ (violet) [13].

The analytic expressions of the inferred potentials $V(r)$ can be found in Refs. [16, 17]. The dependence of $V(r)$ on $r$ (in units of $\mu$ and $\mu^{-1}$, respectively) is shown in Figs. 4 (for $m=0$ ) and 5 (for $m=\mu$ ). Their most striking feature is, due to our ansatz, a logarithmically softened Coulomb singularity at the origin.

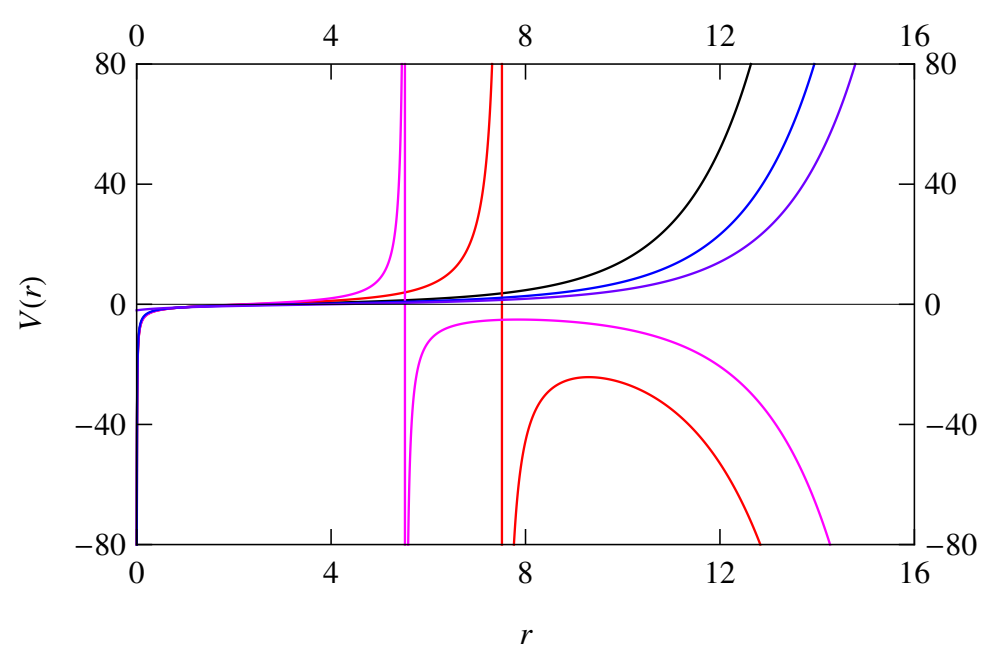

Figure 4. Configuration-space potential $V(r)$ from Fierz-symmetric effective interaction, for massless constituent quarks and mixing parameter $\eta=0[16]$ (black), $\eta=1$ (red), $\eta=2$ (magenta), $\eta=-0.5$ (blue), or $\eta=-1$ (violet). 


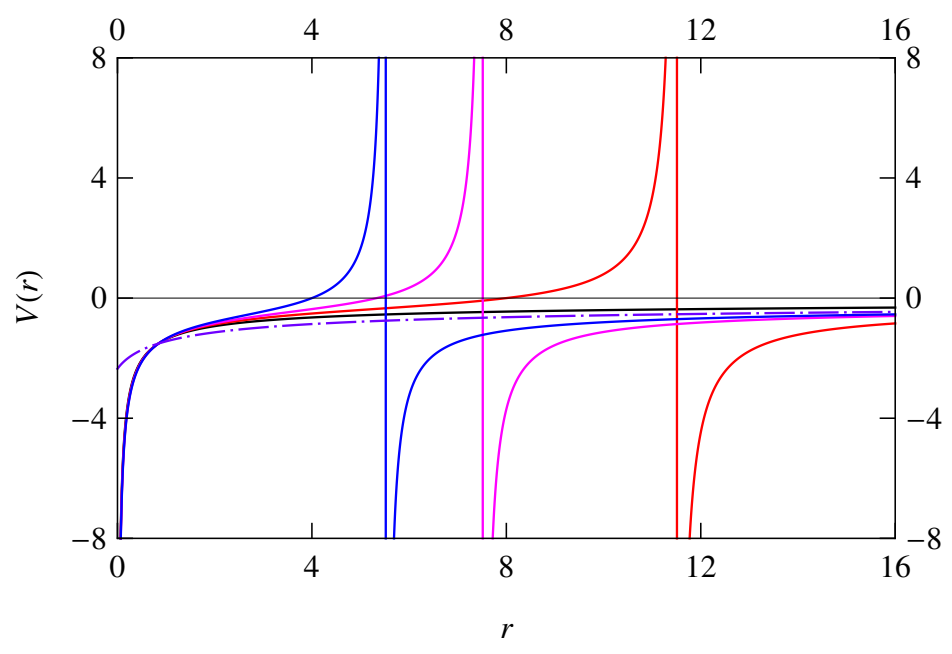

Figure 5. Configuration-space potential $V(r)$ from Fierz-symmetric effective interaction, for constituent quarks of mass $m=\mu$ and mixing value $\eta=0$ [16] (black), $\eta=0.5$ (red), $\eta=1$ (magenta), $\eta=2$ (blue), and $\eta=-1$ (violet).

\section{References}

[1] W. Lucha and F. F. Schöberl, Phys. Rev. D 87 (2013) 016009, arXiv:1211.4716 [hep-ph].

[2] W. Lucha, Proc. Sci., EPS-HEP 2013 (2013) 007, arXiv:1308.3130 [hep-ph].

[3] W. Lucha and F. F. Schöberl, J. Phys. G 31 (2005) 1133, arXiv:hep-th/0507281.

[4] W. Lucha, K. Maung Maung, and F. F. Schöberl, in Proceedings of the International Conference on Quark Confinement and the Hadron Spectrum IV, edited by W. Lucha and K. Maung Maung (World Scientific, Singapore, 2002), p. 340, arXiv:hep-ph/0010078; Phys. Rev. D 63 (2001) 056002, arXiv:hep-ph/0009185; 64 (2001) 036007, arXiv:hep-ph/0011235; W. Lucha and F. F. Schöberl, Int. J. Mod. Phys. A 17 (2002) 2233, arXiv:hep-ph/0109165.

[5] W. Lucha, F. F. Schöberl, and D. Gromes, Phys. Rep. 200 (1991) 127; W. Lucha and F. F. Schöberl, Int. J. Mod. Phys. A 07 (1992) 6431.

[6] W. Lucha and F. F. Schöberl, in Quark Confinement and the Hadron Spectrum VII: $7^{\text {th }}$ Conference on Quark Confinement and the Hadron Spectrum - QCHS7, edited by J. E. F. T. Ribeiro, AIP Conf. Proc. 892 (2007) 524, arXiv:hep-ph/0610016; Li Z.-F., W. Lucha, and F. F. Schöberl, Mod. Phys. Lett. A 21 (2006) 1657, arXiv:hep-ph/0510372.

[7] P. Maris, C. D. Roberts, and P. C. Tandy, Phys. Lett. B 420 (1998) 267, arXiv:nucl-th/9707003.

[8] P. Maris and P. C. Tandy, Phys. Rev. C 60 (1999) 055214, arXiv:nucl-th/9905056.

[9] P. Maris, in Proceedings of the International Conference on Quark Confinement and the Hadron Spectrum IV, edited by W. Lucha and K. Maung Maung (World Scientific, Singapore, 2002), p. 163, arXiv:nucl-th/0009064.

[10] W. Lucha and F. F. Schöberl, preprint HEPHY-PUB 968/16 (2016), arXiv:1606.04781 [hep-ph].

[11] E. E. Salpeter, Phys. Rev. 87 (1952) 328.

[12] P. Maris and C. D. Roberts, Phys. Rev. C 56 (1997) 3369, arXiv:nucl-th/9708029.

[13] W. Lucha and F. F. Schöberl, Phys. Rev. D 93 (2016) 096005, arXiv:1603.08745 [hep-ph]. 
[14] Z.-F. Li, W. Lucha, and F. F. Schöberl, Phys. Rev. D 76 (2007) 125028, arXiv:0707.3202 [hep$\mathrm{ph}]$.

[15] C. D. Roberts, Prog. Part. Nucl. Phys. 61 (2008) 50, arXiv:0712.0633 [nucl-th].

[16] W. Lucha and F. F. Schöberl, Phys. Rev. D 92 (2015) 076005, arXiv:1508.02951 [hep-ph].

[17] W. Lucha and F. F. Schöberl, Phys. Rev. D 93 (2016) 056006, arXiv:1602.02356 [hep-ph]. 